\title{
Body fat, insulin resistance, energy expenditure and serum concentrations of leptin, adiponectin and resistin before, during and after pregnancy in healthy Swedish women
}

\author{
Britt Eriksson ${ }^{1}$, Marie Löf ${ }^{1}$, Hanna Olausson $^{2}$ and Elisabet Forsum $^{1} *$ \\ ${ }^{1}$ Department of Clinical and Experimental Medicine, Faculty of Health Sciences, Linköping University, SE 58185 Linköping, \\ Sweden \\ ${ }^{2}$ Department of Clinical Nutrition, Sahlgrenska Academy, University of Gothenburg, Box 459, SE 40530 Göteborg, Sweden \\ (Received 3 March 2009 - Revised 17 June 2009 - Accepted 29 June 2009 - First published online 25 August 2009)
}

Healthy human pregnancy is associated with changes in food intake, body fatness, energy expenditure and insulin resistance. However, available knowledge is limited regarding the physiological basis of these changes. Published evidence suggests that so-called adipokines (i.e. leptin, adiponectin and resistin) have significant roles when such changes are established. We explored, throughout a complete pregnancy, relationships between total body fat (TBF), energy expenditure, insulin resistance (homeostasic model of insulin resistance, HOMA-IR) and serum concentrations of leptin, adiponectin and resistin. Such concentrations were assessed before pregnancy in gestational weeks $8,14,20,32$ and 35 , and 2 weeks postpartum in twenty-three healthy women. TBF, BMR $(n$ 23) and HOMA-IR $(n$ 17) were assessed before pregnancy in gestational weeks 14 and 32 and 2 weeks postpartum. TBF $(\%)$ was correlated with HOMA-IR $(r 0.68-0.79, P<0.01)$ and with serum leptin $(r$ 0.85$0.88, P<0.001)$ before and during pregnancy. Serum leptin was correlated with HOMA-IR $(r 0.53-0.70, P<0 \cdot 05)$ before and during pregnancy. Serum adiponectin was inversely correlated with HOMA-IR in gestational week $32(r-0 \cdot 52, P<0 \cdot 05)$. When HOMA-IR was regressed on TBF $(\%)$, the slope of the regression line was 0.046 before pregnancy, which was significantly $(P<0 \cdot 05)$ different from the corresponding value, $0 \cdot 111$, in gestational week 32. The results indicate that pregnancy has an enhancing effect on the relationship between body fatness and insulin resistance. This effect, possibly mediated by leptin, may represent a mechanism by which offspring size is regulated in response to the nutritional situation of the mother.

Adipokines: Body fat: Energy expenditure: Insulin resistance: Pregnancy

Healthy human pregnancy is associated with a number of changes in the maternal physiology and metabolism, which are closely linked to the nutritional needs of mother and foetus. However, available knowledge is limited regarding the physiological basis of these changes. In this context, the so-called adipokines (i.e. leptin, adiponectin and resistin) are of interest due to evidence suggesting that these hormones are involved in the regulation of food intake, body fatness, energy expenditure and insulin resistance ${ }^{(1-4)}$, i.e. variables well known to be affected during pregnancy and important in relation to the nutritional situation of women during gestation. A number of studies on pregnant women have been published regarding serum concentrations of these adipokines $^{(5-8)}$ as well as the relationship between such concentrations, on the one hand, and energy expenditure, body fatness and insulin resistance on the other hand. However, as indicated below, many of these studies included women with gestational diabetes, while others were conducted using less appropriate methods to assess body fatness. The value of studies with these limitations may be questionable when the purpose is to understand the physiological and biological basis for the kind of changes during pregnancy that are mentioned above. To adequately assess the impact of the maternal nutritional situation on offspring growth, development and health, such understanding is a requirement.

Serum concentrations of leptin correlate with the total body fat (TBF) content in pregnant ${ }^{(5)}$ and non-pregnant individuals $^{(5,9,10)}$ and with insulin resistance in non-pregnant women $^{(9)}$. A correlation between leptin in serum and insulin resistance has also been found in a group of pregnant women including women with gestational diabetes ${ }^{(11)}$ and in healthy women in the first trimester ${ }^{(12)}$. Results from studies regarding relationships between serum concentrations of adiponectin and body fatness are contradictory in pregnant ${ }^{(6,7,13)}$ and non-pregnant ${ }^{(9,14)}$ women, probably because body fatness is not an independent predictor of adiponectin in serum ${ }^{(14)}$. An inverse correlation between serum adiponectin and insulin resistance has been found in non-pregnant women ${ }^{(9,14,15)}$ and in groups of pregnant women including subjects with gestational diabetes ${ }^{(13,16)}$. With respect to resistin, Silha et al. ${ }^{(9)}$

Abbreviations: HOMA-IR, homeostasic model of insulin resistance; TBF, total body fat. 
found no correlation between body fatness and serum concentrations in non-pregnant women. We found no reports on relationships between resistin in serum and TBF or homeostatic model of insulin resistance (HOMA-IR) in pregnant women.

We previously assessed body fat and energy metabolism in healthy women in a longitudinal study covering a reproductive cycle and using a valid method to assess body composition $^{(17,18)}$. This provided an opportunity to explore relationships before, during and after pregnancy between energy expenditure, $\mathrm{TBF}$, insulin resistance and serum concentrations of leptin, adiponectin and resistin. Results from such a study have not been reported previously.

Body fatness is known to be linked to insulin resistance, and Silha et al. ${ }^{(9)}$ found a significant relationship between TBF and HOMA-IR in non-pregnant women. This relationship is apparently also important during pregnancy, since gestational diabetes is more common in obese than in lean women ${ }^{(19)}$. The design and methodology used in the present study provided an opportunity to study the effect of pregnancy on the relationship between TBF and HOMA-IR.

\section{Materials and methods}

\section{Study outline}

The women arrived at the hospital in the morning for collection of blood samples and for the assessment of body weight, body composition and BMR. Blood sampling and recording of body weight were conducted before pregnancy in gestational weeks $8,14,20,32$ and 35 , and 2 weeks postpartum, while body composition and BMR were assessed before pregnancy in gestational weeks 14 and 32 and 2 weeks postpartum. BMR was assessed using indirect calorimetry as previously described $^{(18)}$. The study was conducted according to the guidelines laid down in the declaration of Helsinki, and all procedures involving human subjects were approved by the Ethics Committee of the University of Linköping, Sweden. Verbal informed consent, witnessed and formally recorded, was obtained from all subjects.

\section{Subjects}

Twenty-three women aged 30 (SD 4) years and planning pregnancy were recruited through the health care system, or by advertising in the local press ${ }^{(18)}$. Before pregnancy, all the women were healthy with normal blood glucose (4.2 (SD 0.5) $\mathrm{mmol} / \mathrm{l})$ and serum insulin (7.4 (SD 2.4) $\mathrm{mU} / \mathrm{l})$ concentrations. Their dietary intake was assessed before pregnancy using three $24 \mathrm{~h}$ dietary recalls as previously described $^{(20)}$. None of the women developed gestational diabetes. Two women had BMI values $>30 \mathrm{~kg} / \mathrm{m}^{2}$ before conception. The women conceived 8-524 $\mathrm{d}$ after the pre-pregnant measurement, and gestational age was calculated on the basis of an ultrasound examination in gestational week 12-14. During pregnancy, they gained 18 (SD 7) $\mathrm{kg}$ of body weight. They all delivered one healthy baby (gestational age 280 (SD 10) d) with a birth weight of 3740 (SD 510) g. Information about birth weight was obtained from hospital records. The women were asked to come to the hospital in a fasting state for all measurements, and upon arrival they were asked when they had last eaten any food. Three women reported not being in a strictly fasting state on one or two measurement occasions. We were able to assess blood glucose and serum insulin in seventeen (the HOMA-IR subgroup) out of the twenty-three women before pregnancy in gestational weeks 14 and 32 and 2 weeks postpartum. Women in this subgroup were all in the fasting state on these measurement occasions.

\section{Body composition}

Body weight of the women was recorded using the KCC150 scale (Mettler-Toledo, Albstadt, Germany). Total body water was assessed using ${ }^{2} \mathrm{H}$ dilution as previously described ${ }^{(17)}$. To calculate fat-free mass, total body water was divided by the appropriate hydration fraction, which was 0.718 before pregnancy, 0.723 at week $14,0.747$ at week 32 and 0.734 at postpartum $^{(17)}$. These figures were assessed in seventeen out of the twenty-three women using data for body weight, total body water and body volume assessed by means of underwater weighing $^{(17)}$. TBF was calculated as body weight minus fatfree mass.

\section{Analyses of blood and serum sample}

Blood was collected from a cubital vein and its glucose content was measured colorimetrically using the HemoCue analyser (HemoCue AB, Ängelholm, Sweden). The blood was kept at $+4^{\circ} \mathrm{C}$ for $4 \mathrm{~h}$ and thereafter centrifuged at $1500 \mathrm{~g}$ for $10 \mathrm{~min}$. Serum was harvested and stored at $-70^{\circ} \mathrm{C}$. Serum insulin was measured by means of RIA (Pharmacia Insulin RIA 100, Pharmacia \& UpJohn Diagostics, Uppsala, Sweden). Insulin resistance (HOMA-IR) was calculated according to Matthews et al. ${ }^{(21)}$. Serum glucose concentration was estimated by multiplying the blood glucose concentration by $1 \cdot 11$. Adipokines were analysed using kits from Linco Research (St Charles, MO, USA) i.e. the human leptin ELISA kit (catalogue no. EZHL-80SK), the human adiponectin ELISA kit (catalogue no. EZHADP-61K) and the human resistin ELISA kit (catalogue no. EZHR-95K). According to the manufacturer, these ELISA kits measure leptin in free and bound forms, all multimeric forms of adiponectin and both hexameric and trimeric forms of resistin, respectively. However, for resistin, it has not been possible to definitely confirm which isoforms the assay actually measures.

\section{Statistics}

Values are given as means and standard deviations. Significant differences were identified using multiple regression analysis with TBF $(\%)$ and individual variables for stage of gestation as indicator variables. Interaction between $\mathrm{TBF}(\%)$ and stage of gestation was used to test whether the effect of TBF (\%) on the dependent variable differed significantly between different stages of gestation. This test was followed by a Bonferroni correction to adjust for multiple comparisons. ANOVA followed by Tukey's test was also used to identify significant differences. Non-normally distributed variables were $\log$ transformed before ANOVA. Linear regression analysis was also used. When neither the dependent nor the independent variable was normally distributed, Spearman's $r$ is given. Otherwise Pearsons's $r$ is reported. Significance was 
accepted when $P<0 \cdot 05$. Statistical analyses were carried out using Statistica software, version 7.1 (StatSOFT, Scandinavia AB, Uppsala, Sweden) or Minitab, version 15 (Minitab Inc., State College, PA, USA).

\section{Results}

\section{Characteristics of women and serum concentration of adipokines}

Table 1 shows body weight, body fat and BMR before, during and after pregnancy for all twenty-three women in the study. The corresponding values for the seventeen women in the HOMA-IR subgroup did not differ in any important way from those shown in Table 1. Table 2 shows the dietary intake of the women before pregnancy. Table 3 shows blood glucose, serum insulin and HOMA-IR before, during and after pregnancy for women in the HOMA-IR subgroup. Table 4 shows serum concentrations of leptin, adiponectin and resistin before, during and after pregnancy for all twenty-three women in the study. These results for individual women are shown in Fig. 1.

\section{Leptin, total body fat, homeostasic model of insulin resistance} and $B M R$

Fig. 2 shows results obtained when leptin in serum was regressed on TBF (\%) before, during and after pregnancy. The ratio between serum leptin and TBF (\%) is also given. $\mathrm{TBF}(\%)$ was found to be significantly correlated with

Table 1. Body weight, total body fat and BMR before and during pregnancy and postpartum for the twentythree women in the study

(Mean values and standard deviations)

\begin{tabular}{|c|c|c|}
\hline & Mean & SD \\
\hline \multicolumn{3}{|l|}{ Before pregnancy } \\
\hline Body weight $(\mathrm{kg})$ & $67 \cdot 2$ & $12 \cdot 0$ \\
\hline Total body fat $(\mathrm{kg})$ & $22 \cdot 6$ & $8 \cdot 9$ \\
\hline Total body fat (\%) & $32 \cdot 7$ & $7 \cdot 8$ \\
\hline $\mathrm{BMI}\left(\mathrm{kg} / \mathrm{m}^{2}\right)$ & $24 \cdot 2$ & $4 \cdot 8$ \\
\hline $\operatorname{BMR}(\mathrm{kJ} / 24 \mathrm{~h})$ & 5430 & 640 \\
\hline \multicolumn{3}{|l|}{ Gestational week 8} \\
\hline Body weight (kg) & $69 \cdot 1$ & $12 \cdot 4$ \\
\hline \multicolumn{3}{|l|}{ Gestational week 14} \\
\hline Body weight $(\mathrm{kg})$ & $69 \cdot 7$ & $12 \cdot 6$ \\
\hline Total body fat $(\mathrm{kg})$ & $24 \cdot 0$ & $8 \cdot 9$ \\
\hline Total body fat (\%) & 33.5 & $7 \cdot 1$ \\
\hline BMR (kJ/24h) & 5580 & 630 \\
\hline \multicolumn{3}{|l|}{ Gestational week 20} \\
\hline Body weight (kg) & $72 \cdot 4$ & $12 \cdot 7$ \\
\hline \multicolumn{3}{|l|}{ Gestational week 32} \\
\hline Body weight (kg) & $79 \cdot 1$ & $13 \cdot 0$ \\
\hline Total body fat $(\mathrm{kg})$ & $27 \cdot 0$ & $9 \cdot 5$ \\
\hline Total body fat (\%) & $33 \cdot 2$ & $7 \cdot 3$ \\
\hline $\operatorname{BMR}(\mathrm{kJ} / 24 \mathrm{~h})$ & 6850 & 970 \\
\hline \multicolumn{3}{|l|}{ Gestational week 35} \\
\hline Body weight (kg) & $81 \cdot 1$ & $13 \cdot 0$ \\
\hline \multicolumn{3}{|l|}{2 weeks postpartum } \\
\hline Body weight $(\mathrm{kg})$ & $73 \cdot 7$ & $13 \cdot 1$ \\
\hline Total body fat $(\mathrm{kg})$ & $26 \cdot 6$ & 8.5 \\
\hline Total body fat (\%) & $35 \cdot 5$ & $6 \cdot 0$ \\
\hline $\operatorname{BMR}(\mathrm{kJ} / 24 \mathrm{~h})$ & 5780 & 850 \\
\hline
\end{tabular}

serum leptin before pregnancy in gestational weeks 14 and 32 as well as postpartum. The equations obtained before pregnancy in gestational weeks 14 and 32 as well as postpartum when serum leptin was regressed on TBF (\%) are also given in Fig. 2. The slopes of these regression lines (2.11-2.66) showed little variation between measurements and were not significantly different. However, the leptin/ TBF ratio increased significantly during pregnancy, especially in gestational week 32. Leptin in serum was significantly correlated with HOMA-IR before pregnancy and in gestational weeks 14 and 32 ( $n$ 17, r 0.53-0.70, $0.002<P<0.05)$, but not postpartum. The increase in TBF (4.4 (SD 3.6) kg, $n$ 23) during the first 32 weeks of pregnancy correlated significantly ( $r \quad 0.58, \quad P<0.01$ ) with the increase in serum leptin (12.7 (SD 16.1) ng/ml, $n$ 23) during this part of pregnancy. Furthermore, the change in TBF between the measurements postpartum and before pregnancy (4.1 (SD 3.8) $\mathrm{kg}, n$ 23) correlated significantly $(r 0.52, P<0.05)$ with the corresponding change in serum leptin $(-4.4$ (SD 14.8) $\mathrm{ng} / \mathrm{ml}$, $n$ 23). The increase in TBF (1.4 (SD 2.6) kg, $n$ 23) during the first 14 weeks of pregnancy did not correlate with the increase in serum leptin (4.6 (SD 11.8) $\mathrm{ng} / \mathrm{ml})$ during this part of gestation. Leptin concentrations in serum were neither correlated with the BMR $(\mathrm{kJ} / 24 \mathrm{~h})$ of the women, nor with $\mathrm{BMR} /$ fat-free mass $(\mathrm{kJ} / 24 \mathrm{~h}$ per $\mathrm{kg})$ at any of the measurement occasions before, during or after pregnancy.

\section{Adiponectin, total body fat and homeostasic model of insulin resistance}

Neither TBF $(\%)$ nor TBF $(\mathrm{kg})$ correlated with adiponectin in serum at any of the measurement occasions before, during or after pregnancy. However, adiponectin in serum was negatively correlated with HOMA-IR in gestational week 32 ( $n$ 17, $r-0.52, P<0.05$ ). Concentrations of adiponectin in serum and of glucose in blood $(\mathrm{mmol} / \mathrm{l})$ were correlated before pregnancy ( $n 17, r-0.57, P<0.05)$, but not during or after pregnancy. The changes in serum adiponectin $(\mu \mathrm{g} / \mathrm{ml})$ were not correlated with the corresponding changes

Table 2. Daily intakes of energy and some nutrients by twenty-three healthy Swedish women before pregnancy

(Mean values and standard deviations)

\begin{tabular}{lrr}
\hline & Mean & SD \\
\hline Energy (kJ) & 8140 & 1540 \\
Protein & & \\
g & 73 & 16 \\
\% of energy intake & 15 & 2 \\
Fat & & \\
g & 71 & 20 \\
$\%$ of energy intake & 32 & 6 \\
Carbohydrates & & 68 \\
$\quad$ g & 249 & 6 \\
$\quad$ of energy intake & 52 & 3 \\
Dietary fibre $(g)$ & 18 & 3 \\
Fe (mg) & 10 & 310 \\
Zn (mg) & 10 & 90 \\
Ca (mg) & 950 & 5 \\
Vitamin D $(\mu \mathrm{g})$ & 5 & \\
Folate $(\mu \mathrm{g})$ & 250 & \\
\hline
\end{tabular}


Table 3. Blood glucose, serum insulin and homeostasic model of insulin resistance (HOMA-IR) before pregnancy in gestational weeks 14 and 32 and 2 weeks postpartum in seventeen Swedish women (the HOMA-IR subgroup)

(Mean values and standard deviations)

\begin{tabular}{|c|c|c|c|c|c|c|c|c|}
\hline & \multicolumn{2}{|c|}{$\begin{array}{c}\text { Before } \\
\text { pregnancy }\end{array}$} & \multicolumn{2}{|c|}{$\begin{array}{l}\text { Gestational } \\
\text { week } 14\end{array}$} & \multicolumn{2}{|c|}{$\begin{array}{c}\text { Gestational } \\
\text { week } 32\end{array}$} & \multicolumn{2}{|c|}{$\begin{array}{c}2 \text { weeks } \\
\text { postpartum }\end{array}$} \\
\hline & Mean & $\mathrm{SD}$ & Mean & $\mathrm{SD}$ & Mean & $\mathrm{SD}$ & Mean & SD \\
\hline Blood glucose $(\mathrm{mmol} / \mathrm{l})$ & $4 \cdot 2$ & 0.5 & $3 \cdot 9^{\star}$ & 0.4 & $3 \cdot 8^{*}$ & 0.5 & $4 \cdot 1$ & 0.4 \\
\hline Serum insulint $(\mathrm{mU} / \mathrm{l})$ & 7.4 & 1.8 & $7 \cdot 6$ & 3.1 & $11 \cdot 0^{*} \ddagger$ & 4.4 & $5 \cdot 1^{*} \neq \S$ & 1.3 \\
\hline HOMA-IR $†$ & 1.6 & 0.5 & 1.5 & 0.6 & $2 \cdot 1 \ddagger$ & $1 \cdot 0$ & $1 \cdot 0^{*} \mp \S$ & 0.3 \\
\hline
\end{tabular}

* Significantly different $(P<0.05)$ from the corresponding value before pregnancy using ANOVA followed by Tukey's test.

$\dagger$ Values were log transformed before ANOVA.

$\ddagger$ Significantly different $(P<0.05)$ from the corresponding value in gestational week 14 using ANOVA followed by Tukey’s test.

$\S$ Significantly different $(P<0.05)$ from the corresponding value in gestational week 32 using ANOVA followed by Tukey's test.

in TBF $(\mathrm{kg})$ during the first 14 or 32 weeks of pregnancy, or with changes between the measurements postpartum and before pregnancy.

\section{Resistin, total body fat and homeostasic model of insulin} resistance

Serum concentrations of resistin did not correlate with TBF $(\%)$ or TBF $(\mathrm{kg})$ either before pregnancy or in gestational weeks 14 or 32 or postpartum. Resistin in serum did not correlate with HOMA-IR ( $n$ 17) either before pregnancy or in gestational weeks 14 or 32 or postpartum. The changes in serum resistin $(\mathrm{ng} / \mathrm{ml})$ were not correlated with the corresponding changes in TBF $(\mathrm{kg})$ during the first 14 or 32 weeks of pregnancy, or between the postpartum measurements and the measurements before pregnancy.

\section{Homeostasic model of insulin resistance and total body fat}

Fig. 3 shows regression analysis with HOMA-IR as the dependent variable and TBF $(\%)$ as the independent variable before pregnancy in gestational weeks 14 and 32 and postpartum. These variables were significantly correlated before pregnancy in gestational weeks 14 and 32, but not postpartum. Furthermore, the slope of the regression line in gestational week 32 $(0 \cdot 111)$ was significantly steeper than the corresponding value $(0 \cdot 046)$ before pregnancy.

\section{Discussion}

Although our subjects were not randomly selected, they were in many respects (BMI before pregnancy ${ }^{(22)}$ and increases in TBF and BMR during pregnancy ${ }^{(23)}$ ) comparable with other Western women. The intake of energy and nutrients of our women was very similar to that reported for other Swedish women of comparable age ${ }^{(24)}$. The dietary intake of our women was also in agreement with that recently recorded for 259 Swedish pregnant women (M Löf, unpublished results). Three women in the present study had ingested a small amount of nutrients before blood sampling. Excluding these women from the analysis did not change the results and conclusions of the present study.

The serum concentrations of leptin and the findings that such concentrations are correlated with body fatness before, during and after pregnancy, as observed in the present study, are consistent with previous results ${ }^{(5,25)}$. However, significant relationships between HOMA-IR and serum leptin before pregnancy as well as in gestational weeks 14 and 32 have not been reported previously in one group of healthy women. Nor have correlations between changes in body fat and changes in serum leptin during pregnancy been identified before. Highman et al. ${ }^{(5)}$ were unable to find such correlations possibly due to the small size of their study. Bajoria et al. ${ }^{\text {(26) }}$ considered that factors other than fat mass alone, such as leptin production by the placenta $^{(4)}$, cause the increased serum leptin concentrations in human pregnancy. This

Table 4. Serum concentrations of leptin, adiponectin and resistin before and during pregnancy and postpartum in twenty-three Swedish women (Mean values and standard deviations)

\begin{tabular}{|c|c|c|c|c|c|c|c|c|c|c|c|c|c|c|}
\hline & \multicolumn{2}{|c|}{$\begin{array}{c}\text { Before } \\
\text { conception }\end{array}$} & \multicolumn{2}{|c|}{$\begin{array}{c}\text { Gestational } \\
\text { week } 8\end{array}$} & \multicolumn{2}{|c|}{$\begin{array}{l}\text { Gestational } \\
\text { week } 14\end{array}$} & \multicolumn{2}{|c|}{$\begin{array}{l}\text { Gestational } \\
\text { week } 20\end{array}$} & \multicolumn{2}{|c|}{$\begin{array}{l}\text { Gestational } \\
\text { week } 32\end{array}$} & \multicolumn{2}{|c|}{$\begin{array}{l}\text { Gestational } \\
\text { week } 35\end{array}$} & \multicolumn{2}{|c|}{$\begin{array}{l}2 \text { weeks } \\
\text { postpartum }\end{array}$} \\
\hline & Mean & SD & Mean & SD & Mean & SD & Mean & SD & Mean & SD & Mean & SD & Mean & SD \\
\hline Leptin & 23.9 & $23 \cdot 6$ & $28 \cdot 1 \dagger$ & $18 \cdot 0$ & $28.6 \dagger$ & $17 \cdot 1$ & $32 \cdot 6 \dagger$ & $18 \cdot 5$ & $36 \cdot 7 \dagger \neq \S$ & $19 \cdot 4$ & $35.8 \dagger$ & $21 \cdot 7$ & $19 \cdot 5 \ddagger \S\|\|^{\star *}$ & $16 \cdot 5$ \\
\hline Adiponectin $(\mu \mathrm{g} / \mathrm{ml})$ & $12 \cdot 6$ & $5 \cdot 8$ & $12 \cdot 0^{\circ}$ & $4 \cdot 2$ & $12 \cdot 4^{\circ}$ & 4.3 & $10 \cdot 9$ & $4 \cdot 3$ & $9 \cdot 0 \dagger \ddagger \S \|$ & $4 \cdot 1$ & $9 \cdot 1 \dagger \ddagger \S \|$ & 4.7 & ৪.0††§॥ & $3 \cdot 2$ \\
\hline Resistin (ng/ml) & $12 \cdot 7$ & 4.1 & $14 \cdot 7$ & $6 \cdot 5$ & $18 \cdot 6 \dagger \ddagger$ & 5.8 & $16 \cdot 0$ & $7 \cdot 1$ & $19 \cdot 8 \dagger \ddagger \|$ & $6 \cdot 6$ & $17.5 \dagger$ & $5 \cdot 8$ & $14 \cdot 7 \S^{\star \star}$ & $6 \cdot 7$ \\
\hline
\end{tabular}

*Values were log transformed before ANOVA.

** Significantly different $(P<0.05)$ from the corresponding value in gestational week 35 using ANOVA and Tukey's test.

† Significantly different $(P<0.05)$ from the corresponding value before pregnancy using ANOVA and Tukey's test.

$\ddagger$ Significantly different $(P<0.05)$ from the corresponding value in gestational week 8 using ANOVA and Tukey's test.

$\S$ Significantly different $(P<0.05)$ from the corresponding value in gestational week 14 using ANOVA and Tukey's test.

\| Significantly different $(P<0.05)$ from the corresponding value in gestational week 20 using ANOVA and Tukey's test.

I Significantly different $(P<0.05)$ from the corresponding value in gestational week 32 using ANOVA and Tukey's test. 

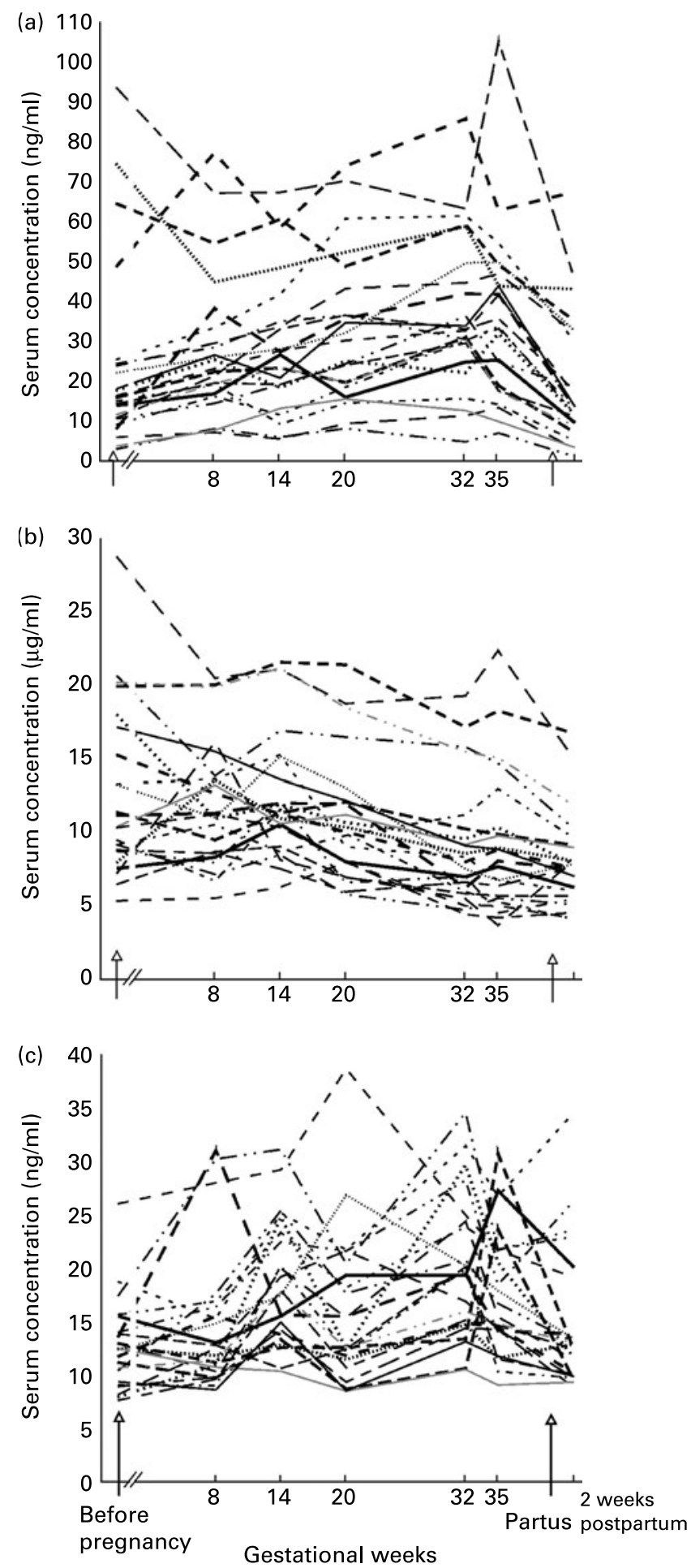

Fig. 1. Serum concentrations of leptin (a), adiponectin (b) and resistin (c) for twenty-three women before pregnancy in gestational weeks 8, 14, 20, 32 and 35 and 2 weeks postpartum. Each subject is represented by one line.

suggestion is supported by the fact that leptin concentrations in serum decrease after delivery. However, Bajoria et al. ${ }^{(26)}$ considered that this suggestion was also supported by the lack of correlations between changes in BMI and serum leptin during pregnancy, and by the finding that during gestation, increases in serum leptin predate the increase in adipose tissue mass. Our data show that serum leptin was already increased in gestational week 8, when only very small increases in body fat can have occurred and when the placenta is not yet fully established. Also, during pregnancy, changes in TBF were correlated with changes in serum leptin. Our findings thus tend to suggest that the increased serum concentration of leptin during pregnancy is not only due to the increased fat mass and leptin production by the placenta, but may also have other as yet unknown explanations. Bajoria et al. ${ }^{(26)}$ and Zavalza-Gómez et al. ${ }^{(4)}$ suggested that a state of leptin resistance develops during pregnancy. We demonstrated that the slopes of regression lines relating $\mathrm{TBF}(\%)$ and leptin in serum showed only small and non-significant differences when comparing values obtained before, during and after pregnancy. These observations do not support the concept of leptin resistance during pregnancy. A well-established role of leptin is its participation in the regulation of energy homeostasis, with effects on food intake and energy expenditure ${ }^{(3)}$. However, we found no relationships between serum concentrations of leptin and BMR. Highman et al. ${ }^{(5)}$ investigated relationships between serum leptin and oxygen consumption before and during pregnancy. Their observations suggested a role for leptin in regulating the changes in energy expenditure occurring during pregnancy. In the present study, we were unable to find support for such a role for leptin.

Our data regarding serum concentrations of adiponectin during pregnancy are in agreement with previous reports ${ }^{(6,7)}$. We have not been able to find any study in healthy pregnant women reporting a negative correlation between serum adiponectin and HOMA-IR. In the present study of women with an insulin resistance typical for healthy women, we found no correlations between TBF and serum adiponectin, or between changes during pregnancy in these two variables, confirming the assertion that body fatness is not an independent predictor of adiponectin in serum ${ }^{(14)}$. Regarding serum concentrations of resistin during pregnancy, the present results were not in complete agreement with previously published data ${ }^{(8,27)}$. We were not able to identify any significant relationships between serum concentrations of resistin, on the one hand, and the TBF content or HOMA-IR, on the other hand, before, during or after pregnancy. Cortelazzi et al. ${ }^{(28)}$ were of the opinion that resistin seems to have a minor role in relation to insulin resistance during pregnancy and our data support this conclusion.

The present results show that body fatness and insulin resistance are related in pregnant and non-pregnant healthy women, and that pregnancy affects the slope of the regression line obtained when HOMA-IR is regressed on TBF (\%). Thus, a woman in the second half of pregnancy tends to be more insulin resistant than a non-pregnant woman with the same TBF content (\%). A high insulin resistance during pregnancy tends to direct glucose to the foetus rather than to maternal tissues. Thus, it may be advantageous for the foetus if the mother gains fat during pregnancy, since this helps it to compete favourably for available nourishment. Note that our data demonstrate this enhancing effect of pregnancy in healthy non-diabetic women. We consider this to be a new and interesting observation. Previous investigators ${ }^{(12)}$ have pointed out that body fat retention may be a causal factor for the 

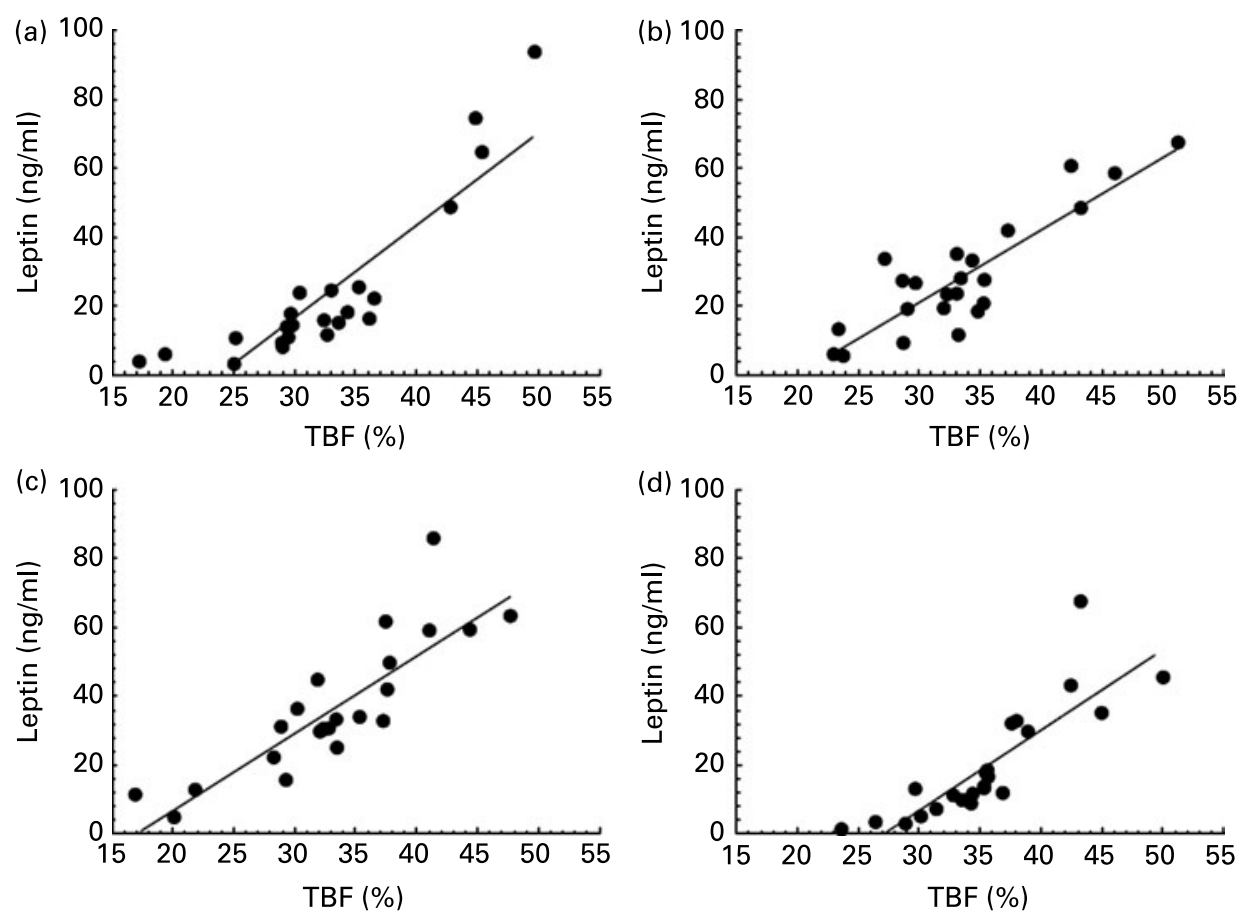

Fig. 2. Relationships between leptin in serum $(\mathrm{ng} / \mathrm{ml})(y)$ and total body fat (TBF) (\%) $(x)$ before pregnancy (a) in gestational weeks 14 (b) and $32(\mathrm{c})$ as well as 2 weeks postpartum (d) in twenty-three Swedish women. The linear regressions are: $y=2.66 x-62.9, r 0.88(P<0.001)(a) ; y=2.11 x-42.1, r 0.87(P<0.001)$ (b); $y=2.26 x-38.4, r 0.85(P<0.001)$ (c); $y=2.33 x-63.0, r 0.85$ ( $P<0.001)$ (d). Leptin (ng/ml)/TBF (\%) was $0.64^{*}$ (SD 0.36; before pregnancy), $0.80+$ (SD 0.35; gestational week 14), 1.04 (SD 0.40; gestational week 32) and 0.50¥ (SD 0.46; 2 weeks postpartum). Using ANOVA followed by Tukey's test, the following significant $(P<0.05)$ differences were found: * different from gestational weeks 14 and 32 ; $†$ different from gestational week 32 and 2 weeks postpartum; $\ddagger$ different from gestational week 32 .
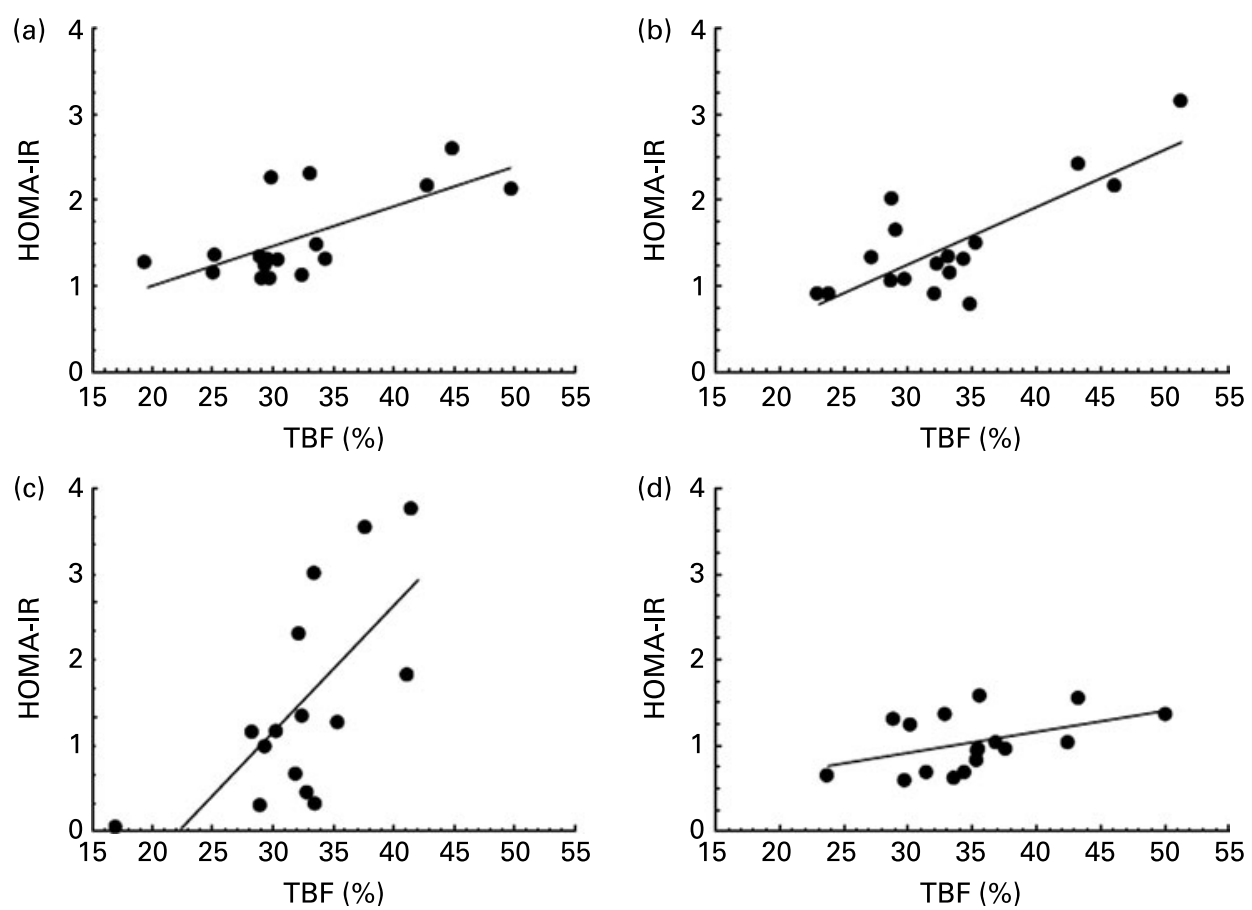

Fig. 3. Relationships between (homeostasis model assessment of insulin resistance HOMA-IR) $(y)$ and total body fat (TBF) (\%) ( $x$ ) before pregnancy (a) in gestational weeks $14(\mathrm{~b})$ and 32 (c) as well as 2 weeks postpartum (d) in seventeen Swedish women. The linear regressions are: $y=0.046 x+0.10, r 0.678(P<0.01)$ (a); $y=0.067 x-0.75, r 0.788(P<0.001)(b) ; y=0.111^{*} x-1.46, r 0.789(P<0.001)$ (c); $y=0.024 x+0.17, r 0.456(P>0.05)(d) ;{ }^{*}$ significantly $(P<0.05)$ different from the corresponding values obtained before pregnancy and 2 weeks postpartum using multiple regression analysis followed by a Bonferroni correction. (HOMA-IR/TBF (\%)) 100 was 4.89† (SD 1.21; before pregnancy), 4.35† (SD 1.22; gestational week 14), 6.37 (SD 2.08; gestational week 32) and 2.93 (SD 0.88; 2 weeks postpartum). Using ANOVA followed by Tukey's test, the following significant $(P<0.05)$ differences were found: $\dagger$ different from gestational week 32 and 2 weeks postpartum; ¥different from 2 weeks postpartum. 
decrease in insulin sensitivity during normal pregnancy and the relationship between body fat and insulin resistance is well established. The new finding in the present paper is that healthy pregnancy enhances this relationship. The mechanism behind this enhancing effect is unknown. Our data suggest that adiponectin may have a role for the physiological increase in insulin resistance during pregnancy. Published data $^{(2)}$ indicate that leptin is also involved when insulin resistance is established. Therefore, it is interesting to note that serum concentrations of leptin correlated with HOMA-IR in our healthy women before and during pregnancy. This information together with the well-established relationship between leptin and body fatness makes it relevant to propose that leptin has a role regarding the enhancing effect of pregnancy on the relationship between body fatness and insulin resistance as identified in the present study.

The present findings can be reconciled with data showing a relationship between the maternal TBF content and birth weight $^{(29)}$ and between the maternal pre-pregnant BMI and birth weight ${ }^{(30)}$. These reports suggest that a high-fat content of the mother stimulates foetal growth. Our observation regarding an enhancing effect of pregnancy on the relationship between body fatness and insulin resistance may represent an important physiological explanation for the strong tendency by pregnant women to retain body fat. It is conceivable that the maternal fat content has a role in the regulation of foetal growth, which could be very useful biologically since fat mothers tend to live in an environment where food is plentiful, while the opposite is true for lean mothers. Body size is an important factor determining the amount of dietary energy required by man and other mammals. We speculate that the present results represent a mechanism by which offspring size is regulated in response to the availability of dietary energy in the environment in which the mother is living.

\section{Acknowledgements}

The present study was designed by E. F. and conducted by M. L. and H. O. B. E. was responsible for the analysis of adipokines and compiled the data in the manuscript. B. E. and E. F. wrote the manuscript that was reviewed by the other authors. The authors' thanks go to all the women in the study, to Anna Christina Granath for help with the analysis of adipokines and to Olle Eriksson for help with statistical analysis. None of the authors had any conflict of interest. The present study was supported by the Swedish Research Council projects no. 12172 and 15402 and the County Council of Östergötland.

\section{References}

1. Arner P (2005) Insulin resistance in type 2 diabetes - role of the adipokines. Curr Mol Med 5, 333-339.

2. Antuna-Puente B, Feve B, Fellahi S, et al. (2008) Adipokines: the missing link between insulin resistance and obesity. Diabetes Metab 34, 2-11.

3. Henry BA \& Clarke IJ (2008) Adipose tissue hormones and the regulation of food intake. J Neuroendocrinol 20, 842-849.

4. Zavalza-Gómez AB, Anaya-Prado R, Rincón-Sánchez AR, et al. (2008) Adipokines and insulin resistance during pregnancy. Diabetes Res Clin Pract 80, 8-15.
5. Highman TJ, Friedman JE, Huston LP, et al. (1998) Longitudinal changes in maternal serum leptin concentrations, body composition, and resting metabolic rate in pregnancy. Am J Obstet Gynecol 178, 1010-1015.

6. Fuglsang J, Skjaerbaek C, Frystyk J, et al. (2006) A longitudinal study of serum adiponectin during normal pregnancy. BJOG 113, 110-113.

7. Mazaki-Tovi S, Kanety H, Pariente C, et al. (2007) Maternal serum adiponectin levels during human pregnancy. J Perinatol 27, 77-81.

8. Palik E, Baranyi E, Melczer Z, et al. (2007) Elevated serum acylated (biologically active) ghrelin and resistin levels associate with pregnancy-induced weight gain and insulin resistane. Diab Res Clin Pract 76, 351-357.

9. Silha JV, Nyomba BL, Leslie WD, et al. (2007) Ethnicity, insulin resistance, and inflammatory adipokines in women at high and low risk for vascular disease. Diabetes Care 30, 286-291.

10. Considine RV (2005) Human leptin: an adipocyte hormone with weight-regulatory and endocrine functions. Semin Vasc Med 5, 15-24.

11. McLachlan KA, O’Neal D, Jenkins A, et al. (2006) Do adiponectin, TNF $\alpha$, leptin and CRP relate to insulin resistance in pregnancy? Studies in women with and without gestational diabetes, during and after pregnancy. Diabetes Metab Res Rev 22, 131-138.

12. Mastorakos G, Valsamakis G, Papatheodorou DC, et al. (2007) The role of adipocytokines in insulin resistance in normal pregnancy: visfatin concentrations in early pregnancy predict insulin sensitivity. Clin Chem 53, 1477-1483.

13. Vitoratos N, Delivelotou A, Vlahos NF, et al. (2008) Serum adiponectin during pregnancy and postpartum in women with gestational diabetes and normal controls. Gyn Endocrinol 24, 614-619.

14. Jürimäe $J$ \& Jürimäe T (2007) Plasma adiponectin concentration in healthy pre- and postmenopausal women: relationship with body composition, bone mineral, and metabolic variables. Am $J$ Physiol Endocrinol Metab 293, E42-E47.

15. Gavrila A, Chan JL, Yiannakouris N, et al. (2003) Serum adiponectin levels are inversely associated with overall and central fat distribution but are not directly regulated by acute fasting or leptin administration in humans: cross-sectional and interventional studies. J Clin Endocrinol Metab 88, 4823-4831.

16. Altinova AE, Toruner F, Bozkurt N, et al. (2007) Circulating concentrations of adiponectin and tumor necrosis factor- $\alpha$ in gestational diabetes mellitus. Gynecol Endocrinol 23, 161-165.

17. Löf M \& Forsum E (2004) Hydration of fat-free mass in healthy women with special reference to the effect of pregnancy. Am J Clin Nutr 80, 960-965.

18. Löf M \& Forsum E (2006) Activity pattern and energy expenditure due to physical activity before and during pregnancy in healthy Swedish women. Br J Nutr 95, 296-302.

19. King JC (2006) Maternal obesity, metabolism, and pregnancy outcomes. Annu Rev Nutr 26, 271-291.

20. Löf M \& Forsum E (2004) Validation of energy intake by dietary recall against different methods to assess energy expenditure. J Hum Nutr Dietet 17, 471-480.

21. Matthews DR, Hosker JP, Rudenski AS, et al. (1985) Homeostasis model assessment: insulin resistance and ß-cell function from fasting plasma glucose and insulin concentrations in man. Diabetologia 28, 412-419.

22. Bostrom G (2001) Chapter 6. Habits of life and public health. The National Public Health Report 2001. Scand J Public Health 29, Suppl. 58, 156-166.

23. United Nations University, World Health Organization, Food and Agricultural Organization of the United Nations (2004) Human Energy Requiremets. Joint FAO/WHO/UNU Expert Consultation. FAO Technical Report Series no. 1, pp. 53-62. Geneva: WHO. 
24. Becker W \& Pearson M (2002) Riksmaten 1997-98. Kostvanor och näringsintag $i$ Sverige. Metod och resultatanalys. Uppsala, Sweden: National Food Administration.

25. Butte NF, Hopkinson JM \& Nicolson MA (1997) Leptin in human reproduction: serum leptin levels in pregnant and lactating women. J Clin Endocrinol Metab 82, 585-589.

26. Bajoria R, Sooranna SR, Ward BS, et al. (2002) Prospective function of placental leptin at maternal-fetal interface. Placenta 23, 103-115.

27. Chen D, Dong M, Fang Q, et al. (2005) Alterations of serum resistin in normal pregnancy and pre-eclampsia. Clin Sci 108, 81-84.
28. Cortelazzi D, Corbetta S, Ronzoni S, et al. (2007) Maternal and foetal resistin and adiponectin concentrations in normal and complicated pregnancies. Clin Endocrinol 66, 447-453.

29. Forsum E, Löf M, Olausson H, et al. (2006) Maternal body composition in relation to infant birth weight and subcutaneous adipose tissue. Br J Nutr 96, 408-414.

30. Baker JL, Michaelsen KF, Rasmussen KM, et al. (2004) Maternal prepregnant body mass index, duration of breastfeeding, and timing of complementary food introduction are associated with infant weight gain. Am J Clin Nutr 80, $1579-1588$. 